\title{
Computational Assessment of Novel Predicted Compounds in Ni- Re Alloy System
}

\author{
Siya $\mathrm{Zhu}^{1}{ }^{1} \cdot$ Axel van de Walle ${ }^{1}$
}

Submitted: 25 January 2021 /in revised form: 1 April 2021 / Accepted: 2 April 2021 / Published online: 15 April 2021

(C) The Author(s) 2021

\begin{abstract}
Ab initio high-throughput efforts are continuously identifying new intermetallic compounds in a wide range of alloy systems that were previously thought to be well-characterized. While such predictions are likely valid near absolute zero, they carry the risk that such phases become unstable at the higher temperature relevant to typical synthesis conditions. We illustrate how this possibility can be rapidly tested by integrating Calphad modeling into the high-throughput loop. As an example, we investigate the $\mathrm{Ni}-\mathrm{Re}$ system, in which $\mathrm{D} 0_{19}$ and $\mathrm{D} 1_{\mathrm{a}}$ phases were predicted as possible intermetallic compounds. We confirm that these phases are indeed stable at practical synthesis temperatures and explain how they could have been overlooked in prior assessments.
\end{abstract}

Keywords ab Initio methods - computational studies . nickel $\cdot$ phase diagram $\cdot$ rhenium

\section{Introduction}

Ni-Re alloys have high technological relevance for aerospace applications. Rhenium, with a high melting point $3459 \mathrm{~K}^{[1]}$ and desirable mechanical properties, ${ }^{[2]}$ is widely used as refractory materials in aerospace applications such as rocket nozzles. ${ }^{[3,4]}$ Some of its alloys enjoy similar properties and, in particular, the Ni-Re alloy shows good strength properties at high temperature. ${ }^{[5]}$

Siya Zhu

siya_zhu@brown.edu

1 Brown University School of Engineering, Providence
Levy et al. ${ }^{[6]}$ made a number of predictions of novel intermetallic compounds in Re-based alloys, including two possible new phases in the Ni-Re system. They used highthroughput DFT based calculations and found a $\mathrm{D}_{19}$ $\left(\mathrm{NiRe}_{3}\right)$ and a $\mathrm{D} 1_{\mathrm{a}}\left(\mathrm{Ni}_{4} \mathrm{Re}\right)$ to be ground states. This discovery, however, appears at odds with prior experimental thermodynamic assessments, ${ }^{[7-10]}$ which find no evidence of intermetallic phases. We thus revisit this finding using recent high-throughput computational methods based on the Calphad framework combined with the Special Quasirandom Structures $(\mathrm{SQS})^{[11]}$ formalism. These methods incorporate finite-temperature thermodynamics and hence enable us to clarify the situation.

\section{Method}

Our thermodynamic model is based on the Compound Energy Formalism (CEF). ${ }^{[12]}$ Accordingly, the phases with a simple lattice (i.e., FCC and HCP) are modelled jointly with the corresponding superstructures (here $\mathrm{D} 1_{\mathrm{a}}$ and $\mathrm{D} 0_{19}$, respectively).

In this work, we use the sqs2tdb tools of the Alloy Theoretic Automated Toolkit (ATAT) ${ }^{[13,14]}$ to generate all structural input files and to construct the Calphad model from the ab initio calculations output. For structures not included in ATAT's pre-generated database of SQS, the "mcsqs" $\operatorname{code}^{[15]}$ is used to generate suitable SQS with controlled compositions on each sublattice. This independent control of the sublattice composition enables us to easily determine all the parameters of the CEF models. Vibrational entropy contributions are included for all end members. However, interaction terms (between and across sublattices) are considered temperature independent. Configurational entropy is modeled, as is traditionally done for 
CEF models, using an ideal solution model on each sublattice. The free energy of pure FCC Ni and HCP Re are taken from the SGTE elemental database ${ }^{[16]}$ while the HCP-FCC difference is obtained from our first-principles calculations.

For the first-principles calculations, the Vienna Ab-initio Simulation Package (VASP) ${ }^{[17-20]}$ is used with the PerdewBurke-Ernzerhof (PBE) exchange and correlation functional at level of the generalized gradient approximation (GGA). ${ }^{[21]}$ A cutoff energy of $500 \mathrm{eV}$ is used in the projector augmented wave (PAW) method. ${ }^{[22]} \mathrm{K}$-points per reciprocal atom is set as 8000 for all calculations. For the phonon calculations, a Born von Karman model is used ${ }^{[23]}$ and was constructed with the fitfc code ${ }^{[15]}$ with force constant range up to $3 \AA$.

For completeness, we also consider the liquid phase. We use the SQS for liquid provided in the ATAT database as a starting geometry and run first-principles molecular dynamics with NVT ensemble for the average energy. Temperature is set as $41 \mathrm{~K}$ above the melting temperature for pure $\mathrm{Ni}$ and $\mathrm{Re}$, and set with linear relationship with concentration for mixtures of $\mathrm{Ni}$ and $\mathrm{Re}$. This provides formation enthalpies as a function of composition, while the formation entropy is obtained from an ideal solution model. The solid-liquid free energies differences for the elements are taken from the SGTE elemental database. ${ }^{[16]}$

\section{Results}

$\mathrm{D}_{19}$ phase belongs to the space group $\mathrm{P}_{3} / \mathrm{mmc}$ (\#194), with stoichiometry $\mathrm{NiRe}_{3}$. It divides HCP structure into two sublattices, with all rhenium atoms occupy one set of lattice and nickel atoms locate at the center of icosahedrons formed by 12 rhenium atoms (Fig. 1).

Since the SQS's of $\mathrm{D}_{19}$ structure are not included in the ATAT database, we use Monte Carlo method to generate SQS's of $\mathrm{D}_{19}$ with "mcsqs" module in ATAT. In Fig. 2 we show the SQS's with different concentrations of both sublattices.

With these SQS's we get the disordered structures of $\mathrm{D}_{19}$ phase Ni-Re. After that structures are optimized, and free energies are calculated (Table 1). Phonon contributions to the entropy $\left(\mathrm{S}_{\mathrm{vib}}\right)$ are also included for the endmembers, in the high-temperature (ht) limit.

$\mathrm{D} 1_{\mathrm{a}}$ phase belongs to the space group I4/m (\#87) with stoichiometry $\mathrm{Ni}_{4} \mathrm{Re}$. It divides simple cubic lattice into two sets of sublattices. Ni atoms occupy one set of sublattice, and Re atoms occupy another set (Fig. 3).

Like D019, we build the SQS's of D1 a phase (Fig. 4) and calculate the free energies and vibration entropies of endmembers (Table 2).

Table 3 reports the liquid formation enthalpies given by molecular dynamics calculations. These data are used to fit the temperature-independent interaction parameter $(16664 \mathrm{~J} / \mathrm{mol})$ of regular solution model of the formation free energy. The free energy difference between the solid and liquid phase of the pure end members are taken from the SGTE database.

With all the structures built and thermodynamic stats calculated, we use PANDAT to plot a phase diagram of NiRe (Fig 5). We also plot some data points from previous experimental investigation ${ }^{[7,24-29]}$ for comparison.

\section{Discussion}

It is first important to note that this approach only requires a relatively small number of structural free energy calculations, thus making it ideally suited to act as a screening step for future high throughput ordered structure predictions. Even the phonon calculations are kept at a very manageable cost because they only involve high-symmetry end members. The liquid state calculations are the most expensive but are clearly not essential for the purpose of assessing ordered phases.

Our thermodynamic model captures the asymmetry on the solubility limits (with the HCP phase being wider than the FCC one). However, our thermodynamic model appears to over-predict the high temperatures mutual solubility of $\mathrm{Ni}$ in $\mathrm{Re}$ and vice versa, relative to the most recent experimental assessments, ${ }^{[7]}$ although this discrepancy, when measured along the energy dimension is actually fairly small: A change in free energy by about 2

Fig. 1 Structure of $\mathrm{D}_{19}$ phase $\mathrm{NiRe}_{3}$. Grey (light) balls are rhenium atoms and blue (dark) balls are nickel atoms
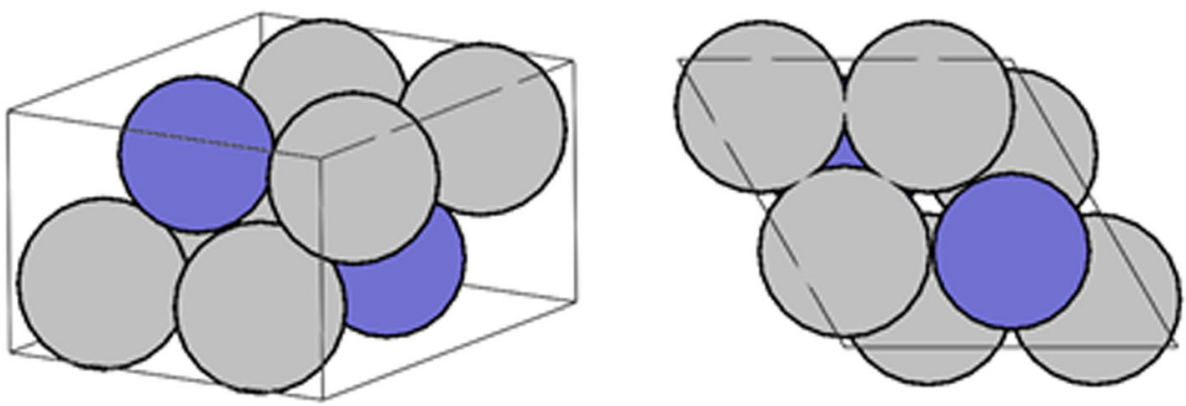
Fig. 2 SQS's with different concentrations of both sublattice for $\mathrm{D} 0_{19}$ phase. White balls are A sublattice and black balls are B sublattice. Balls with and without shadows stand for different occupations of two elements in one sublattice. (a) $\mathrm{X}^{\mathrm{A}}=1, \mathrm{X}^{\mathrm{B}}=1$, each sublattice is occupied by one kind of atoms; (b) $\mathrm{X}^{\mathrm{A}}=0.5, \mathrm{X}^{\mathrm{B}}=1, \mathrm{~A}$ sublattice is occupied randomly with both kinds of atoms with concentrations 0.5 of each. B sublattice is occupied by only one kind of atoms; (c) $\mathrm{X}^{\mathrm{A}}=1$, $\mathrm{X}^{\mathrm{B}}=0.5$, A sublattice is occupied by only one kind of atoms, while B sublattice is occupied randomly with both atoms with concentrations 0.5 of each; (d) $\mathrm{X}^{\mathrm{A}}=\mathrm{X}^{\mathrm{B}}=0.5$, both sublattices are occupied randomly with both kinds of atoms with concentration 0.5 of each

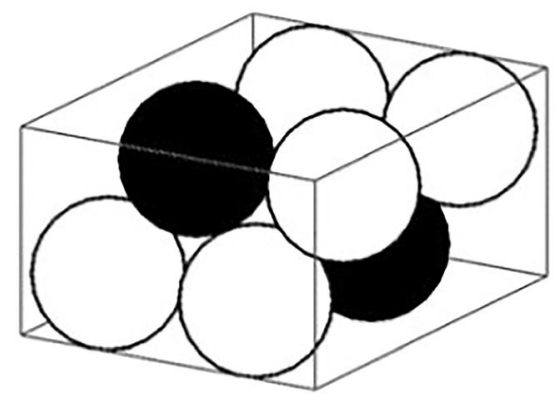

(a)

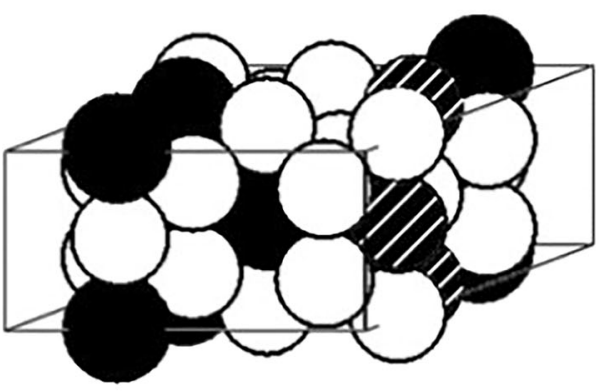

(c)

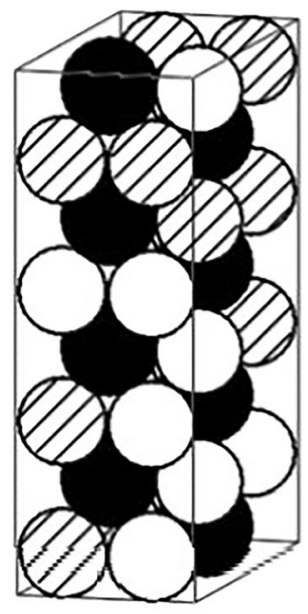

(b)

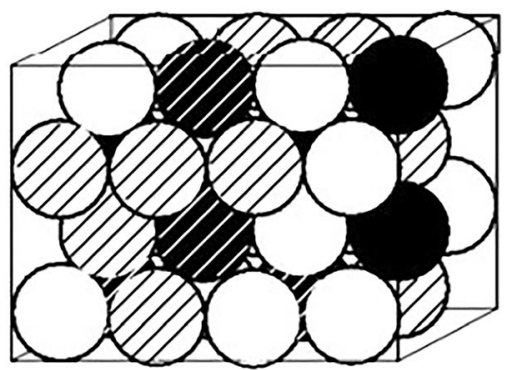

(d)

Table 1 Free energies at $0 \mathrm{~K}$ of $\mathrm{DO}_{19} \mathrm{Ni-Re}$ and $\mathrm{S}_{\mathrm{vib} \_ \text {ht }}$ of endmembers

\begin{tabular}{|c|c|c|c|c|}
\hline $\begin{array}{l}\text { Concentration of Re in sublattice } \\
\qquad \mathrm{A} \mathrm{X}_{\mathrm{Re}}^{\mathrm{A}}\end{array}$ & $\begin{array}{l}\text { Concentration of Re in sublattice } \\
\qquad \mathrm{B} \mathrm{X}_{\mathrm{Re}}^{\mathrm{B}}\end{array}$ & $\begin{array}{l}\text { \# of atoms in a } \\
\text { cell }\end{array}$ & $\begin{array}{l}\text { Free energy per cell at } 0 \mathrm{~K} \\
(\mathrm{eV})\end{array}$ & $\begin{array}{l}\mathrm{S}_{\text {vib_ht }}(\text { Boltzmann } \\
\text { Constant })\end{array}$ \\
\hline \multirow[t]{3}{*}{0} & 0 & 8 & -44.374 & -704.504 \\
\hline & 0.5 & 32 & -205.202 & $\ldots$ \\
\hline & 1 & 8 & -57.611 & -701.902 \\
\hline \multirow[t]{3}{*}{0.5} & 0 & 32 & -259.315 & $\ldots$ \\
\hline & 0.5 & 32 & -288.049 & $\ldots$ \\
\hline & 1 & 32 & -314.782 & $\ldots$ \\
\hline \multirow[t]{3}{*}{1} & 0 & 8 & -86.549 & -701.012 \\
\hline & 0.5 & 32 & -370.746 & $\ldots$ \\
\hline & 1 & 8 & -99.386 & -695.312 \\
\hline
\end{tabular}

$\mathrm{kJ} / \mathrm{mol}$ would be sufficient to bring the two sets of results in agreement. Our phase diagram also shows an allotropic transition for pure Re at high temperature, which has never been observed in experiments. This is most likely an artifact of using a simple harmonic model for the phonon free energy, which neglects the effect of thermal expansion. Note once again that FCC and HCP Re have a difference in free energy of only $1.56 \mathrm{~kJ} / \mathrm{mol}$ at $3000{ }^{\circ} \mathrm{C}$ in our calculation, which is small. In addition, our results give a higher peritectic temperature of $2044{ }^{\circ} \mathrm{C}$. These discrepancies are not entirely unexpected given that our approach is intended to push the accuracy-speed trade-off in favor of speed. Our methods might provide a lower accuracy for the prediction of phase boundaries (especially the liquid phase) compared with experimental studies ${ }^{[30]}$ but enable the efficient prediction of the stability of novel candidate 
Fig. 3 Structure of $D 1_{a}$ phase $\mathrm{Ni}_{4}$ Re. Grey (light) balls are rhenium atoms, and blue (dark) balls are nickel atoms
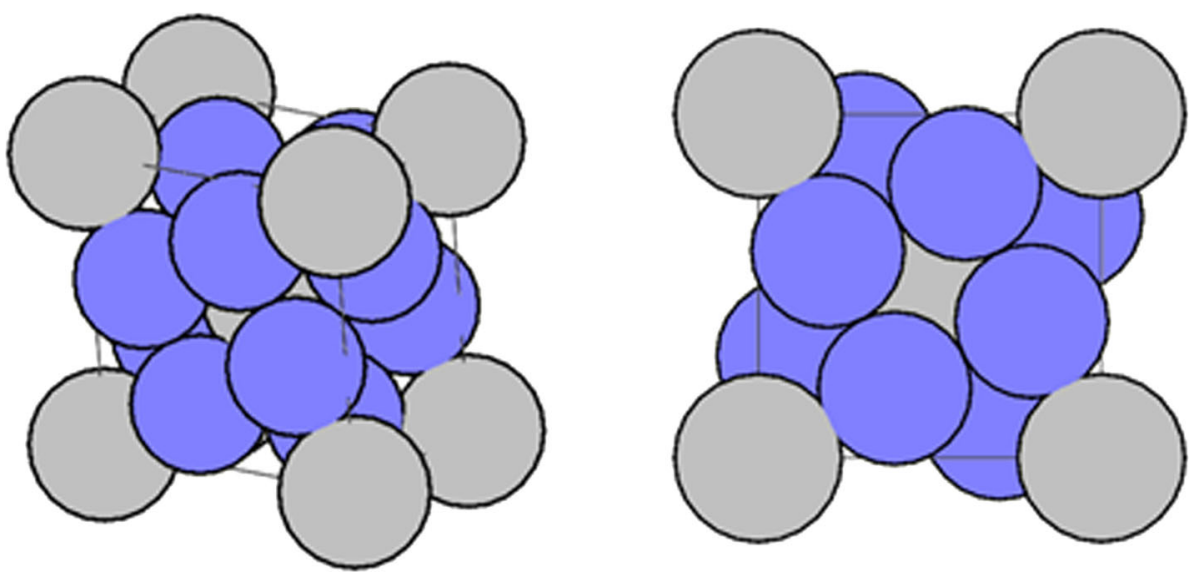

Fig. 4 SQS's with different concentrations of both sublattice for $\mathrm{D} 1_{\mathrm{a}}$ phase. White balls are $\mathrm{A}$ sublattice and black balls are B sublattice. Balls with and without shadows stand for different occupations of two elements in one sublattice. (a) $\mathrm{X}^{\mathrm{A}}=1, \mathrm{X}^{\mathrm{B}}=1$, each sublattice is occupied by one kind of atoms; (b) $\mathrm{X}^{\mathrm{A}}=0.5, \mathrm{X}^{\mathrm{B}}=1, \mathrm{~A}$ sublattice is occupied randomly with both kinds of atoms with concentrations 0.5 of each. B sublattice is occupied by only one kind of atoms; (c) $\mathrm{X}^{\mathrm{A}}=1$, $\mathrm{X}^{\mathrm{B}}=0.5$, A sublattice is occupied by only one kind of atoms, while B sublattice is occupied randomly with both atoms with concentrations 0.5 of each; (d) $X^{\mathrm{A}}=\mathrm{X}^{\mathrm{B}}=0.5$, both sublattices are occupied randomly with both kinds of atoms with concentration 0.5 of each

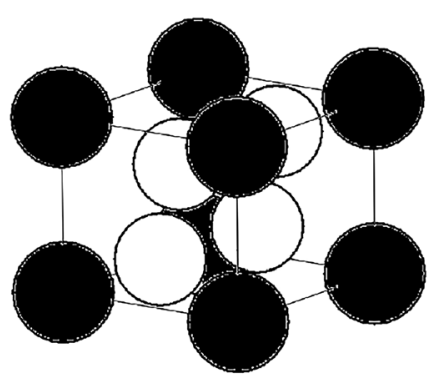

(a)

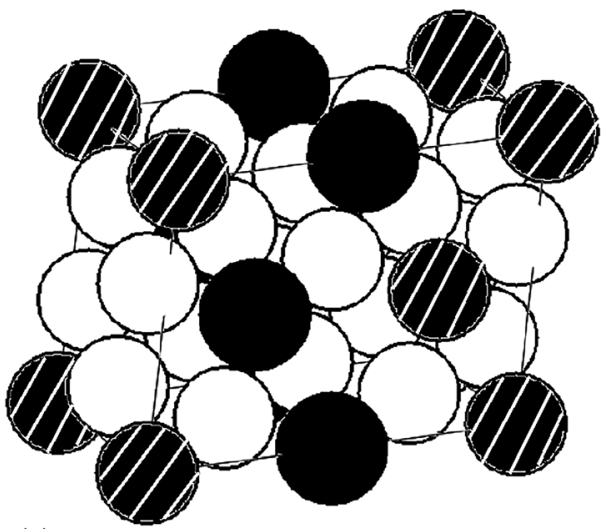

(c)

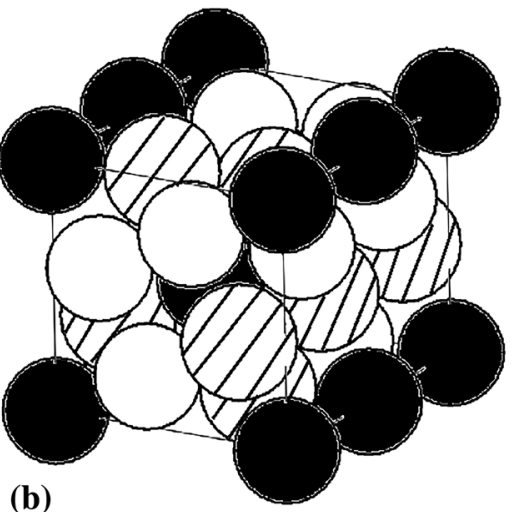

(b)

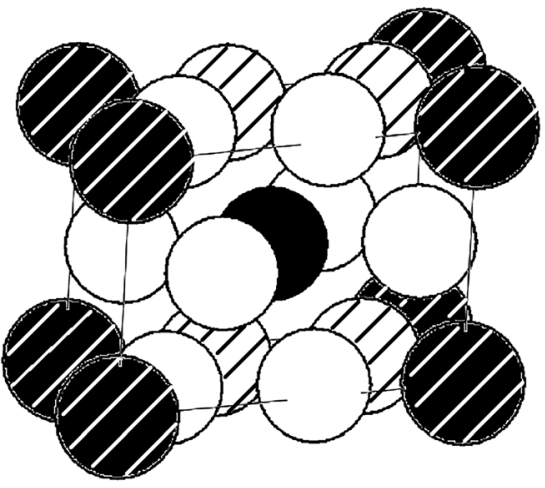

(d) phases and the general topology of phase diagrams. Still, it is highly suggestive that multiple older experimental studies do also find that the solubility limit of the hep phase field could extends to near 50\%. ${ }^{[24,25]}$

Most importantly, our results corroborate the stability of the new ordered phases over a temperature range relevant to accessible synthesis conditions. At the same time, the calculated disordering temperature are sufficiently low that it is highly plausible that the ordered phases could have been missing in earlier experimental investigations. In particular, of all the phase boundary measurements, the lowest temperature considered is $990^{\circ} \mathrm{C}^{[27]}$, which is still higher than the highest stable temperature of ordered new phases by our calculations. A few older measurements ${ }^{[24]}$ do go down to $600^{\circ} \mathrm{C}$ on the Ni-rich side and suggest a much lower solubility limit for $\mathrm{Re}$ in $\mathrm{Ni}$ than would be suggested by extrapolating other measurements made at higher temperatures, which is probably one reason why they were ignored in recent assessments. Our results suggest a natural explanation for this discrepancy: The lower temperature data points perhaps represent equilibria with the ordered $\mathrm{D} 1_{\mathrm{a}}$ phase rather than the hcp phase, thus abruptly reducing the Re solubility. A likely reason for the absence of experimental observation of the novel phases 
Table 2 Free energies at $0 \mathrm{~K}$ of $\mathrm{D} 1_{\mathrm{a}} \mathrm{Ni}-\mathrm{Re}$ and $\mathrm{S}_{\mathrm{vib} \_ \text {ht }}$ of endmembers

\begin{tabular}{|c|c|c|c|c|}
\hline $\begin{array}{l}\text { Concentration of Re in sublattice } \\
\qquad \mathrm{A} \mathrm{X}_{\mathrm{Re}}^{\mathrm{A}}\end{array}$ & $\begin{array}{l}\text { Concentration of Re in sublattice } \\
\qquad B X_{\operatorname{Re}}{ }^{B}\end{array}$ & $\begin{array}{l}\text { \# of atoms in a } \\
\text { cell }\end{array}$ & $\begin{array}{c}\text { Free energy per cell at } \\
0 \mathrm{~K}, \mathrm{eV}\end{array}$ & $\begin{array}{l}\mathrm{S}_{\mathrm{vib} \_\mathrm{ht}}(\text { Boltzmann } \\
\text { Constant })\end{array}$ \\
\hline \multirow[t]{3}{*}{0} & 0 & 5 & -27.862 & -440.580 \\
\hline & 0.5 & 20 & -125.228 & $\ldots$ \\
\hline & 1 & 5 & -34.997 & -440.718 \\
\hline \multirow[t]{3}{*}{0.5} & 0 & 20 & -165.920 & $\ldots$ \\
\hline & 0.5 & 20 & -179.084 & $\ldots$ \\
\hline & 1 & 20 & -192.807 & $\ldots$ \\
\hline \multirow[t]{3}{*}{1} & 0 & 5 & -55.091 & -435.061 \\
\hline & 0.5 & 20 & -233.564 & $\ldots$ \\
\hline & 1 & 5 & -61.816 & -433.320 \\
\hline
\end{tabular}

Table 3 Calculated energies of liquid Ni-Re

\begin{tabular}{lccc}
\hline Concentration of $\operatorname{Re}\left(\mathrm{X}_{\mathrm{Re}}\right)$ & Melting Point, $\mathrm{K}$ & MD temperature, $\mathrm{K}$ & Energy per atom, $\mathrm{eV}$ \\
\hline 0 & 1728 & 1769 & -5.44428 \\
0.25 & $\ldots$ & 2202 & -7.04828 \\
0.5 & $\ldots$ & 2635 & -8.70988 \\
0.75 & $\ldots$ & 3067 & -10.3583 \\
1 & 3459 & 3500 & -12.0444 \\
\hline
\end{tabular}

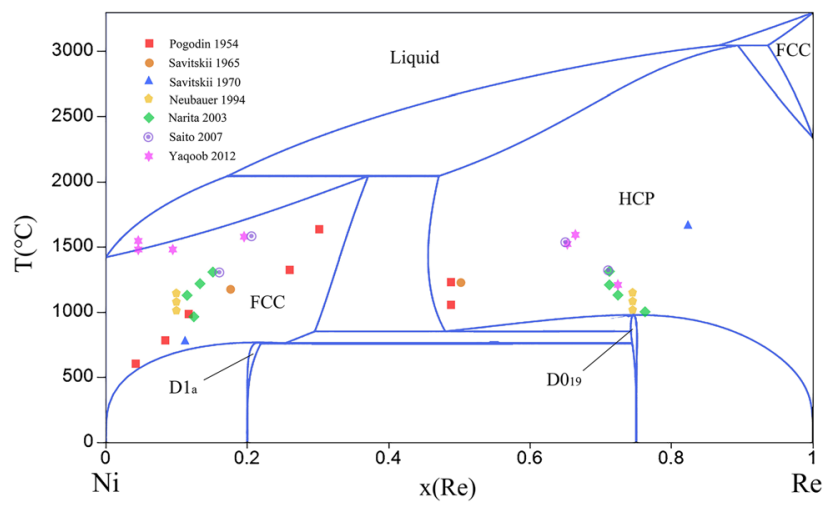

Fig. 5 Phase diagram of Ni-Re plotted with our calculated data and experimental investigations

are the difficulty in achieving thermodynamic equilibrium experimentally at low temperature, due to slow kinetics and potential difficulties in resolving small precipitates.

The primary goal of the proposed approach is to rapidly verify if predicted ground states warrant further consideration in a full assessment of a given phase diagram. In this work, we have found it more efficient to model the ordered phases jointly with their corresponding disordered parent lattices. This means that the CEF model, as generated here, should be interpreted as the "projection" of the true alloy thermodynamic onto the CEF representation that best approximates the free energy of the alloy. The resulting free energy are thus reliable even though the site fractions in the disordered phase may not be, as is apparent form the fact that the site fractions are not equal. It should be noted that these deviations from equal site fractions only amount to at most $5 \%$ on the dominant sublattice and $20 \%$ on the minority sublattice. For completeness, we include the TDB file resulting from our fitting process, although we would not recommend that it be used as is to construct a CALPHAD assessment, due to the approximate treatment of the SRO in the fcc and hcp phases. We have attempted to include the fcc and hcp phases as single-sublattice models (with SRO effects properly included via a CVM-based method, ${ }^{[13]}$ ) however the resulting free energies are nearly degenerate with the multi-sublattice descriptions used here and manually correcting the multiple spurious crossing of the corresponding free energies or integrating the singleand multiple-lattice descriptions into a single CEF model would lead to a method that is not really amenable to a high-throughput treatment.

\section{Conclusion}

In this work, we combine the SQS and CEF formalisms to build a thermodynamic model of the Ni-Re system. This enables us to re-assess prior predictions of two possible new phases: $\mathrm{D}_{19}$ and $\mathrm{D} 1_{\mathrm{a}}$ in this system. While we corroborate their stability at lower temperature, we find relative low disordering temperatures which explains why they 
may have been missed by experimental assessments. This case study illustrates that our method provides effective means to screen for the relevance of novel phase predictions in other systems via high-throughput calculations. It remains of interest to build upon these methods to achieve a higher accuracy in the predicted phase boundaries, especially at high temperature.

Acknowledgments The authors acknowledge support from the National Science Foundation through grant DMR-2001411. Computational resources were provided by the Center for Computation and Visualization at Brown University and the Extreme Science and Engineering Discovery Environment (XSEDE) Stampede2 at the Texas Advanced Computing Center through allocation TGDMR050013N, which is supported by National Science Foundation Grant No. ACI-1548562.

Open Access This article is licensed under a Creative Commons Attribution 4.0 International License, which permits use, sharing, adaptation, distribution and reproduction in any medium or format, as long as you give appropriate credit to the original author(s) and the source, provide a link to the Creative Commons licence, and indicate if changes were made. The images or other third party material in this article are included in the article's Creative Commons licence, unless indicated otherwise in a credit line to the material. If material is not included in the article's Creative Commons licence and your intended use is not permitted by statutory regulation or exceeds the permitted use, you will need to obtain permission directly from the copyright holder. To view a copy of this licence, visit http://creativecommons. org/licenses/by/4.0/.

\section{References}

1. Y. Zhang, J.R. Evans, and S. Yang, Corrected Values for Boiling Points and Enthalpies of Vaporization of Elements in Handbooks, J. Chem. Eng. Data, 2011, 56(2), p 328-337

2. M. de Jong, J. Kacher, M. Sluiter, L. Qi, D. Olmsted, A. Van de Walle, J. Morris Jr., A. Minor, and M. Asta, Electronic Origins of Anomalous Twin Boundary Energies in Hexagonal Close Packed Transition Metals, Phys. Rev. Lett., 2015, 115(6), p 065501

3. D. L. Mittendorf and T. G. Sutton, Method for making a rhenium rocket nozzle. 1999, Google Patents.

4. A. Naumov, Rhythms of Rhenium, Russ. J. Non-Ferrous Met., 2007, 48(6), p 418-423

5. J. Schooling and P. Reed, The application of neural computing methods to the modelling of fatigue in Ni-base superalloys. 1996.

6. O. Levy, M. Jahnátek, R.V. Chepulskii, G.L. Hart, and S. Curtarolo, Ordered Structures in Rhenium Binary Alloys from FirstPrinciples Calculations, J. Am. Chem. Soc., 2011, 133(1), p $158-163$

7. K. Yaqoob, and J.-M. Joubert, Experimental Determination and Thermodynamic Modeling of the Ni-Re Binary System, J. Solid State Chem., 2012, 196, p 320-325

8. H. Okamoto, $\mathrm{Ni-Re}$ (nickel-rhenium). Journal of phase equilibria, 1992. 13(3).
9. H. Okamoto, Ni-Re (Nickel-Rhenium), J. Phase Equilib. Diffus., 2012, 33(4), p 346-346

10. A. Nash, and P. Nash, Ni-Re (Nickel-Rhenium) System, Bull. Alloy Phase Diagrams, 1985, 6(4), p 348-350

11. A. Zunger, S.-H. Wei, L. Ferreira, and J.E. Bernard, Special Quasirandom Structures, Phys. Rev. Lett., 1990, 65(3), p 353

12. M. Hillert, The Compound Energy Formalism, J. Alloys Compd., 2001, 320(2), p 161-176

13. A. van de Walle, R. Sun, Q.-J. Hong, and S. Kadkhodaei, Software Tools for High-Throughput CALPHAD from First-Principles Data, Calphad, 2017, 58, p 70-81

14. A. Van De Walle, M. Asta, and G. Ceder, The alloy theoretic automated toolkit: A user guide. arXiv preprint cond-mat/ 0212159, 2002.

15. A. Van De Walle, Multicomponent Multisublattice Alloys, Nonconfigurational Entropy and Other Additions to the Alloy Theoretic Automated Toolkit, Calphad, 2009, 33(2), p 266-278

16. A.T. Dinsdale, SGTE Data for Pure Elements, Calphad, 1991, 15(4), p 317-425

17. G. Kresse, and J. Hafner, Ab Initio Molecular Dynamics for Liquid Metals, Phys. Rev. B, 1993, 47(1), p 558

18. G. Kresse, and J. Hafner, Ab Initio Molecular-Dynamics Simulation of The Liquid-Metal-Amorphous-Semiconductor Transition in Germanium, Phys. Rev. B, 1994, 49(20), p 14251

19. G. Kresse, and J. Furthmüller, Efficiency of Ab-Initio Total Energy Calculations for Metals and Semiconductors Using a Plane-Wave Basis Set, Comput. Mater. Sci., 1996, 6(1), p 15-50

20. G. Kresse, and J. Furthmüller, Efficient Iterative Schemes for Ab Initio Total-Energy Calculations Using a Plane-Wave Basis Set, Phys. Rev. B, 1996, 54(16), p 11169

21. J.P. Perdew, K. Burke, and M. Ernzerhof, Generalized Gradient Approximation Made Simple, Phys. Rev. Lett., 1996, 77(18), p 3865

22. P.E. Blöchl, Projector Augmented-Wave Method, Phys. Rev. B, 1994, 50(24), p 17953

23. A. van de Walle, and G. Ceder, Automating First-Principles Phase Diagram Calculations, J. Phase Equilib., 2002, 23(4), p 348

24. S. Pogodin, and M. Skryabina, Nickel-Rhenium System, Isz Sekt Fiz-Khim Analiza, 1954, 25, p 81-88

25. E. Savitskii, M. Tylkina, and K. Povarova, Rhenium Alloys [in Russian]. 1965.

26. E. Savitskii and G. Burkhanov, Alloys of Refractory Metals, in Physical Metallurgy of Refractory Metals and Alloys. 1970, Springer. p. 191-234.

27. C. Neubauer, D. Mari, and D. Dunand, Diffusion in the NickelRhenium System, Scr. Metall. Mater., 1994, 31(1), p 99-104

28. S. Narita, Master's thesis. Graduate School of Hokkaido University, 2003.

29. S. Saito, K. Kurokawa, S. Hayashi, T. Takashima, and T. Narita, Phase Equilibria and Tie-Lined Compositions in a Ternary Ni-AlRe System at 1423 K, J. Jpn. Inst. Met., 2007, 71(9), p 793-800

30. W.J. Boettinger, D.E. Newbury, N. Ritchie, M.E. Williams, U.R. Kattner, E. Lass, K.-W. Moon, M.B. Katz, and J. Perepezko, Solidification of Ni-Re Peritectic Alloys, Metall. and Mater. Trans. A., 2019, 50(2), p 772-788

Publisher's Note Springer Nature remains neutral with regard to jurisdictional claims in published maps and institutional affiliations. 